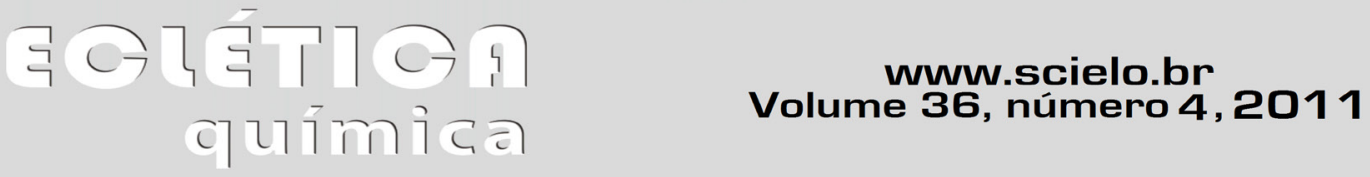

\title{
DETERMINATION OF ORGANOCHLORINE PESTICIDE RESIDUES IN HUMAN MILK IN PIRACICABA, SP
}

\author{
Rosana M.O. FREGUGLIA*1; Graziela C. R. MOURA ANDRADE; Valdemar Luiz TORNISIELO. \\ ${ }^{1}$ Laboratório de Ecotoxicologia, Centro de Energia Nuclear na Agricultura - CENA/USP, Av. Centenário, 303, Bairro São Dimas, Caixa \\ Postal 96, CEP: 13400-970, Piracicaba - SP.* autor correspondente: ro_freguglia@hotmail.com
}

Worldwide, there is great concern with the issue of bioaccumulation of organochlorine compounds in human milk and their potential impact on health. Breastfeeding is recognized as essential for the proper development and protection of children's health. In this study, we collected human milk samples from 40 donors in Piracicaba - SP, and analyzed to determine the levels of residues of organochlorine pesticides: hexachlorobenzene (HCB), lindane, heptachlor, aldrin, heptachlor epoxide, $o, p$ ' DDE, a- endosulfan, $p, p$ ' DDE, dieldrin, endrin, b-endosulfan, endosulfan sulfate, and methoxychlor. The analysis was performed by solid phase extraction (SPE) and quantification by gas chromatography using electron capture detector (ECD). Residues of organochlorine pesticides were detected in $65 \%$ of the donors evaluated.

\section{Introdução}

O uso indiscriminado de pesticidas e a potencial contaminação ambiental associada têm preocupado pesquisadores e órgãos governamentais, principalmente os envolvidos com as questões de saúde pública e meio ambiente. O mau uso dos pesticidas pode acarretar efeitos nocivos tais como: aumento do número de pragas resistentes; toxicidade crônica e aguda em animais, inclusive ao homem (contaminação e intoxicação); mortalidade de algumas espécies; poluição ambiental [1] e resíduos em alimentos .

Devido a sua eficiência no controle de pragas, os pesticidas organoclorados foram intensivamente empregados na agricultura desde a década de 1940. A partir da década de 1970, devido a seus efeitos carcinogênicos e acumulativos, seu uso foi restringido. A presença de resíduos de organoclorados no ambiente está diretamente associada as propriedades físico-químicas deste grupo de moléculas, tais como natureza lipofílica, estabilidade e persistência [2].

O leite humano é uma via de eliminação dos pesticidas organoclorados, devido a sua bioacumulação em tecidos ricos em lipídeos nos organismos, por não ser uma técnica invasiva vem sendo amplamente utiliza- do para avaliação da concentração desses compostos em adultos (fêmeas em idade fértil), bem como a exposição das crianças [3]. Os lactantes consomem quantidades substanciais de leite materno que podem atingir ou exceder a ingestão diária aceitável destes compostos [4].

Laug et al. [5], foram os primeiros pesquisadores a relatar a presença de resíduos de DDT em amostras de leite humano nos Estados Unidos. A partir deste estudo, novas investigações em diferentes regiões do globo também detectaram resíduos de pesticidas organoclorados em amostras de leite humano, inclusive em comunidades isoladas e localizadas em áreas remotas [6]. Apesar do uso de alguns dos compostos da classe dos organoclorados estar atualmente proibido pela legislação brasileira [7], resíduos destes compostos ainda são comumente encontrados, persistindo no meio ambiente, podendo assim contaminar águas, alimentos, animais e pessoas.

Matuo [8] determinou resíduos do DDT e seus metabólitos em leite humano em doadoras da região de Ribeirão Preto, SP. Os valores encontrados foram comparáveis aos níveis observados em estudos conduzidos em diversos países desenvolvidos, variando entre 0,2 a 6,38 vezes o valor do limite de resíduos estabelecido para leite bovino pela FAO/WHO [9]. Matuo et al. [10], determinaram a presença de resíduos de lindane, heptacloro, aldrin, dieldrin e endrin em adição ao DDT, 
em 37 amostras de leite humano. Os autores relataram a presença de resíduos em porcentagem significativa das amostras analisadas, sendo que o lindane foi detectado em $32 \%$ das amostras, algumas com valores elevados enquanto o heptacloro foi detectado em $65 \%$ das amostras consideradas.

Com a crescente preocupação com o destino e impacto dos pesticidas a FAO/WHO tem formado comissões de especialistas em pesticidas para avaliar o impacto destes compostos, estabelecendo limites de resíduos em alimentos a partir dos dados científicos disponíveis. A ingestão diária aceitável (IDA) expressa em $\mathrm{mg} \mathrm{kg}{ }^{-1}$ de massa corporal, estabelecida por longa avaliação toxicológica em animais experimentais, é definida como a quantidade de uma substância química que pode ser ingerida diariamente pelo homem durante toda vida, sem risco apreciável a sua saúde [11-12].

O presente trabalho teve por objetivo determinar os níveis de resíduos de pesticidas organoclorados hexaclorobenzeno (HCB), lindane, heptacloro, aldrin, heptacloro epóxi, o,p' DDE, a- endosulfan, p,p' DDE, dieldrin, endrin, b-endosulfan, endosulfan sulfato e metoxicloro em leite humano, na região de Piracicaba-SP e, com base nestas determinações, gerar um banco de dados sobre a contaminação de leite humano por resíduos de organoclorados.

\section{Procedimentos Experimentais}

As amostras de leite humano foram obtidas de mães voluntárias em processo de lactação no período de setembro de 2000 a agosto de 2002 . Foram coletados cerca de $30 \mathrm{~g}$ de leite, em frascos de vidro de boca larga. Os frascos foram identificados, acondicionados em caixas térmicas com gelo e transportados ao Laboratório de Ecotoxicologia do Centro de Energia Nuclear na Agricultura - CENA/USP, em Piracicaba - São Paulo. No laboratório, foram conservados sob refrigeração a $4^{\circ} \mathrm{C}$ até o momento da análise.

Foram rastreados os seguintes organoclorados: hexaclorobenzeno (HCB), lindane, heptacloro, aldrin, heptacloro epóxi, o,p' DDE, a- endosulfan, p,p' DDE, dieldrin, endrin, b-endosulfan, endosulfan sulfato e metoxicloro (ChemService, West Chester, USA).

Os solventes utilizados no processo de extração foram: metanol grau resíduo (Tedia Company - EUA); hexano grau resíduo (Mallinckrodt - França). Para lavagem e limpeza da vidraria foi utilizada acetona p.a (J.T. Baker - México) e acetona grau resíduo (Mallinckrodt - França). Foi preparada solução estoque na con- centração $20 \mathrm{hg} \mathrm{mL}^{-1}$ do padrão contendo mistura dos pesticidas organoclorados, em hexano grau resíduo. A partir da solução estoque, foram preparadas soluções de trabalho (mix de organoclorados) nas concentrações: 0,01, 0,02, 0,03, 0,04, 0,05 $\mathrm{hg} \mathrm{mL}^{-1}$ diluidas em hexano.

O método de extração utilizado foi o descrito por Mañes et al. [13] através de extração em fase sólida (SFE) utilizando cartuchos $\mathrm{C}_{18}$ (Sep-Pak Vac - 1g, Waters Corporation) com capacidade de $6 \mathrm{~mL}$. As extrações (filtração e eluição) das amostras foram feitas com auxilio de câmara de vácuo.

Primeiramente, a coluna $\mathrm{C}_{18}$ foi condicionada com $10 \mathrm{~mL}$ de metanol e $10 \mathrm{~mL}$ de água destilada (sob vácuo - fluxo 1 a $2 \mathrm{~mL} \mathrm{~min}^{-1}$ ). Depois foi adicionado 5 $\mathrm{mL}$ de leite, $5 \mathrm{~mL}$ de água destilada, $10 \mathrm{~mL}$ de metanol e após foi homogeneizado por sonificação por $10 \mathrm{~min}$. Após a coluna foi lavada com água destilada (2 vezes) e a água descartada. A eluição foi realizada utilizando 10 $\mathrm{mL}$ de hexano, o extrato foi concentrado e ressuspendido em hexano.

\section{Sistema CG-ECD}

As análises cromatográficas foram realizadas empregando-se um cromatográfo gasoso, marca Chrompack CP 9001, equipado com detector de captura de elétrons ${ }^{63} \mathrm{Ni}$; injetor "split" e Nitrogênio ultrapuro como gás de arraste.

\section{Condições Cromatográficas}

Foi empregada coluna DB-5MS (J\&W Scientific Inc.) com temperatura inicial de $240^{\circ} \mathrm{C}$, final $280^{\circ} \mathrm{C}$, com aquecimento de $20^{\circ} \mathrm{C} \mathrm{min}{ }^{-1}$, com temperatura do injetor de $250^{\circ} \mathrm{C}$ e do detector a $300^{\circ} \mathrm{C}$; e volume de injeção de $2 \mathrm{~mL}$.

Para identificação e confirmação das amostras positivas, foi utilizado cromatógrafo gasoso acoplado ao espectrômetro de massa CG/EM - Finnigan CGQ, coluna DB-5MS (J\&W Scientific Inc.).

A quantificação foi realizada por padronização externa. Para a construção das curvas analíticas foram preparadas cinco soluções de trabalho diluídas com hexano, nas concentrações de $0,01,0,02,0,03,0,04,0,05$ hg $\mathrm{mL}^{-1}$. As amostras de leite foram fortificadas com os organoclorados nas concentrações de 0,01 a $0,05 \mathrm{hg}$ $\mathrm{mL}^{-1}$; homogeneizadas e colocadas a $4^{\circ} \mathrm{C}$ "overnight". $\mathrm{O}$ branco de reagente foi preparado sem a matriz e a testemunha foi preparada com leite integral, sem fortificação.

Os resultados foram avaliados segundo Codex Alimentarius - Resíduos de pesticidas em alimentos/ Limites máximos de resíduos estranhos - LMRE [9] utilizando-se a recomendação de Ingestão Diária Aceitável 
(IDA), expresso em $\mathrm{mg} \mathrm{kg}^{-1}$ de massa corporal, estabelecida por longa avaliação toxicológica em animais experimentais.

A ingestão diária do contaminante (TID) expressa em mg $\mathrm{kg}^{-1}$ de massa corporal foi calculada através da fórmula [9]:

\section{$\mathrm{TID}=\mathrm{C} . \mathrm{F} . \mathrm{M}$}

onde:

$\mathrm{C}=$ Concentração média do contaminante na gordura do leite $\left(\mathrm{mg} \mathrm{g}^{-1}\right)$

$\left.\mathrm{g}^{-1}\right)$

$\mathrm{F}=$ concentração média da gordura no leite ( $\mathrm{g}$

$$
\mathrm{M}=\text { Consumo médio de leite }\left(\mathrm{g} \mathrm{kg}_{\text {dia }}{ }^{-1}\right)
$$

A diferença entre a TID e a IDA foi usada como indicativo para avaliar os níveis de contaminação:

TID - IDA $\leq 0$ : níveis de contaminação abaixo do estabelecido;

TID - IDA >0: níveis acima do estabelecido.

De acordo com Kelts e Jones [14], o valor adotado para consumo médio foi de $125 \mathrm{~g}$ de leite $\mathrm{kg}_{\text {dia }}{ }^{-1}$, o teor de gordura no leite de $3,2 \%$ e densidade de 1,032 $\mathrm{g} \mathrm{mL}^{-1}$.

\section{Validação do método}

A linearidade é a habilidade de um método analítico em produzir resultados diretamente proporcionais à concentração do analito nas amostras em dada faixa de concentração. A linearidade pode ser obtida por padronização interna ou externa e é formulada como expressão matemática usada para o cálculo da concentração do analito a ser determinado na amostra real. O coeficiente de determinação $\left(\mathrm{r}^{2}\right)$ é freqüentemente usado para indicar o quanto pode ser considerada adequada a reta como modelo matemático [15].

O limite de detecção (LD) representa a menor concentração da substância em exame que pode ser detectada, mas não necessariamente quantificada, utilizando um determinado procedimento experimental. Neste estudo foi comparado os picos de amostras em baixas concentrações conhecidas do composto de interesse na matriz e no branco (matriz isenta do composto de interesse), estabelecendo assim uma concentração mínima na qual a substância pode ser facilmente detectada. A relação sinal-ruído considerada foi de 3:1 [16].

O limite de quantificação (LQ) representa a menor concentração da substância em exame que pode ser medida, utilizando um determinado procedimento experimental. Os mesmos critérios de LD foram adotados para o LQ, utilizando a relação 10:1 [16].

A precisão representa a dispersão de resultados entre ensaios independentes, repetidos de uma mesma amostra, amostras semelhantes ou padrões, sob condições definidas e pode ser expressa através da estimativa do desvio padrão relativo (RSD), também conhecido como coeficiente de variação (CV) [16].

A exatidão é definida como sendo a concordância entre o resultado de um ensaio e o valor de referência aceito como convencionalmente verdadeiro [15] e foi avaliada através de ensaios de recuperação.

\section{Resultados}

Os parâmetros de validação do método estão apresentados na Tabela 1 e os valores de recuperação variaram entre 70 e $120 \%$ e os CV foram menores que $20 \%$ para todos os organoclorados rastreados, de acordo com os critérios estabelecidos para análise de resíduos.

Resíduos de pesticidas organoclorados foram detectados em $65 \%$ das 40 amostras de leite humano avaliadas. Do total de amostras avaliadas, 14 não apresentaram níveis detectáveis dos organoclorados monitorados.

Tabela 1. Coeficiente de determinação, média das recuperações (nos níveis 0,02, 0,03 e 0,04 hg $\mathrm{mL}^{-1}$ ), coeficiente de variação, limite de detecção e limite de quantificação

\begin{tabular}{|l|c|c|c|c|c|}
\hline \multicolumn{1}{|c|}{ Organoclorado } & $\mathbf{r}^{2}$ & Rec\% & $\mathbf{C . V . \%}$ & $\begin{array}{c}\mathbf{L D} \\
\eta \mathrm{g} \mu \mathrm{L}^{-1}\end{array}$ & $\begin{array}{c}\mathbf{L Q} \\
\eta \mathrm{g} \mu \mathrm{L}^{-1}\end{array}$ \\
\hline Hexaclorobenzeno & 0,9977 & 103 & 1 & 0,003 & 0,009 \\
\hline Lindane & 0,9939 & 92 & 2 & 0,003 & 0,009 \\
\hline Heptacloro & 0,9930 & 99 & 2 & 0,003 & 0,009 \\
\hline Aldrin & 0,9976 & 80 & 1 & 0,003 & 0,009 \\
\hline Heptacloro epóxi & 0,9968 & 103 & 2 & 0,003 & 0,009 \\
\hline Q. $D^{\prime}$ DDE & 0,9960 & 97 & 1 & 0,003 & 0,009 \\
\hline Endosulfan I & 0,9923 & 95 & 3 & 0,003 & 0,009 \\
\hline D.R' DDE & 0,9979 & 96 & 1 & 0,003 & 0,009 \\
\hline Dieldrin & 0,9973 & 93 & 1 & 0,003 & 0,009 \\
\hline Endrin & 0,9980 & 94 & 3 & 0,003 & 0,009 \\
\hline Endosulfan II & 0,9993 & 91 & 2 & 0,003 & 0,009 \\
\hline Endosulfan sulfato & 0,9989 & 87 & 2 & 0,003 & 0,009 \\
\hline Metoxicloro & 0,9999 & 98 & 1 & 0,003 & 0,009 \\
\hline
\end{tabular}


Tabela 2 - Limite máximo de resíduo estranho, resíduos, ingestão diária aceitável, TID e TID - IDA dos organoclorados detectados no

leite humano

\begin{tabular}{|c|c|c|c|c|c|c|c|c|}
\hline Amostra & Profissão & Lactação & Organoclorado & $\begin{array}{c}\text { LMRE } \\
\left(\mathrm{mg} \cdot \mathrm{kg}^{-1}\right)\end{array}$ & $\begin{array}{l}\text { Resíduo } \\
\left(\mathrm{mg} \cdot \mathrm{kg}^{-1}\right)\end{array}$ & $\begin{array}{c}\text { IDA } \\
\left(\mathrm{mg}^{-1} \mathrm{~kg}^{-1}\right)\end{array}$ & $\begin{array}{c}\text { TID } \\
\left(m g \cdot \mathrm{kg}^{-1}\right)\end{array}$ & $\begin{array}{l}\text { TID - IDA } \\
\left(\mathrm{mg}^{\mathrm{k}} \mathrm{kg}^{-1}\right)\end{array}$ \\
\hline \multirow{2}{*}{ E04 } & \multirow{2}{*}{ Professora } & \multirow[t]{2}{*}{1} & Heptacloro epóxi & 0,006 & 0,0119 & 0,0001 & 0,000040 & $-0,000060$ \\
\hline & & & Dieldrin & 0,006 & 0,0123 & 0,0001 & 0,000043 & $-0,000057$ \\
\hline E17 & Eng. Agro & 1 & Heptacloro epóxi & 0,006 & 0,0063 & 0,0001 & 0,000022 & $-0,000078$ \\
\hline \multirow[t]{2}{*}{ E19 } & \multirow[t]{2}{*}{$\mathrm{PD}^{\mathrm{a}}$} & & Heptacloro epóxi & 0,006 & 0,0350 & 0,0001 & 0,000123 & 0,000023 \\
\hline & & & Dieldrin & 0,006 & 0,0123 & 0,0001 & 0,000043 & $-0,000057$ \\
\hline \multirow[t]{2}{*}{ E20 } & \multirow{2}{*}{$\mathrm{PD}^{\mathrm{a}}$} & & Heptacloro epóxi & 0,006 & 0,0165 & 0,0001 & 0,000060 & $-0,000040$ \\
\hline & & & Dieldrin & 0,006 & 0,0155 & 0,0001 & 0,000056 & $-0,000044$ \\
\hline E21 & $E D^{b}$ & 1 & Heptacloro epóxi & 0,006 & 0,0070 & 0,0001 & 0,000025 & $-0,000075$ \\
\hline E27 & Faxineira & 2 & Heptacloro epóxi & 0,006 & 0,0189 & 0,0001 & 0,000069 & $-0,000031$ \\
\hline \multirow{3}{*}{ E36 } & \multirow{3}{*}{ Autônoma } & & Heptacloro epóxi & 0,006 & 0,0189 & 0,0001 & 0,000069 & $-0,000031$ \\
\hline & & & Dieldrin & 0,006 & 0,0177 & 0,0001 & 0,000064 & $-0,000036$ \\
\hline & & & Hexaclorobenzeno & 0,010 & 0,0376 & 0,0001 & 0,000136 & 0,000036 \\
\hline \multirow{2}{*}{ NE2 } & \multirow{2}{*}{ Química } & & Heptacloro epóxi & 0,006 & 0,0177 & 0,0001 & 0,000064 & \\
\hline & & & Dieldrin & 0,006 & 0,0107 & & 0,000 & $-0,0$ \\
\hline NE15 & Secretária & 1 & Heptacloro epóxi & 0,006 & 0,0063 & 0,0001 & 0,000023 & $-0,000077$ \\
\hline NE16 & Eng. Agro & 1 & Heptacloro epóxi & 0,006 & 0,0061 & 0,0001 & 0,000022 & $-0,000078$ \\
\hline NE05 & Tec. Lab. & 3 & Heptacloro epóxi & 0,006 & 0,0058 & 0,0001 & 0,000021 & $-0,000079$ \\
\hline NE06 & $\mathrm{MS}^{\mathrm{c}}$ & 1 & Heptacloro epóxi & 0,006 & 0,0233 & 0,0001 & 0,000084 & $-0,000016$ \\
\hline \multirow{2}{*}{ NE08 } & \multirow{2}{*}{ A. Adm. ${ }^{d}$} & & Heptacloro epóxi & \multirow{2}{*}{0,006} & 0,0371 & \multirow[t]{2}{*}{0,0001} & 0,000275 & 0,000175 \\
\hline & & & Heptacloro & & 0,0760 & & 0,000135 & 0,000035 \\
\hline Amostra & Profissão & Lactação & Organoclorado & $\begin{array}{c}\text { LMRE } \\
\left(\mathrm{mg} \cdot \mathrm{kg}^{-1}\right)\end{array}$ & $\begin{array}{l}\text { Resíduo } \\
\left(\mathrm{mg} \mathrm{kg}^{-1}\right)\end{array}$ & $\begin{array}{c}\text { IDA } \\
\left(\mathrm{mg} \cdot \mathrm{kg}^{-1}\right)\end{array}$ & $\begin{array}{c}\text { TID } \\
\left(\mathrm{mg} \cdot \mathrm{kg}^{-1}\right)\end{array}$ & $\begin{array}{l}\text { TID - IDA } \\
\left(\mathrm{mg} \cdot \mathrm{kg}^{-1}\right)\end{array}$ \\
\hline NE09 & & 2 & Heptacloro epóxi & 0,006 & 0,0084 & 0,0001 & 0,000031 & $-0,000069$ \\
\hline NEUY & utonoma & 2 & Heptacloro & & 0,0053 & & 0,000019 & $-0,000081$ \\
\hline NE23 & Dentista & 1 & Heptacloro & 0,006 & 0,006 & 0,0001 & & $-0,000$ \\
\hline & & & Dieldrin & 0,006 & 0,0114 & 0,0001 & 0,000041 & $-0,000059$ \\
\hline NE24 & Secretária & 1 & Heptacloro epóxi & 0,006 & 0,0077 & 0,0001 & 0,000028 & $-0,000072$ \\
\hline & & & Hexaclorobenzeno & 0,010 & 0,0129 & 0,0001 & 0,000047 & $-0,000053$ \\
\hline NE25 & Estudante & 1 & Heptacloro epóxi & 0,006 & 0,0085 & 0,0001 & 0,000030 & $-0,000070$ \\
\hline & & & Dieldrin & 0,006 & 0,0037 & 0,0001 & 0,000129 & 0,000029 \\
\hline NE10 & Professora & 1 & Heptacloro & 0,006 & 0,0162 & 0,0001 & 0,000059 & $-0,000041$ \\
\hline & & & Heptacloro & 0,006 & & 0,0001 & & \\
\hline NE26 & C. Super ${ }^{e}$ & 2 & Dieldrin & 0,006 & 0,0546 & 0,0001 & 0,000198 & 0,000098 \\
\hline & & & Hexaclorobenzeno & 0,010 & 0,0226 & 0,0001 & 0,000082 & $-0,000018$ \\
\hline E03 & Vendedora & 1 & Dieldrin & 0,006 & 0,0096 & 0,0001 & 0,000034 & $-0,000066$ \\
\hline NE38 & Dentista & 2 & Heptacloro & 0,006 & & 0,0001 & 0,000059 & $-0,000041$ \\
\hline & & & Hexaclorobenzeno & 0,010 & 0,0158 & 0,0001 & 0,000057 & $-0,000043$ \\
\hline E07 & $P D^{a}$ & 2 & Dieldrin & 0,006 & 0,0153 & 0,0001 & 0,000055 & $-0,000045$ \\
\hline & & & Hexaclorobenzeno & 0,010 & 0,0245 & 0,0001 & 0,000089 & $-0,000011$ \\
\hline E12 & Professora & 1 & Dieldrin & 0,006 & 0,0096 & 0,0001 & 0,000034 & $-0,000066$ \\
\hline NE8 & Aux. Adm. & 2 & Dieldrin & 0,006 & 0,0138 & 0,0001 & 0,000050 & $-0,000050$ \\
\hline NE01 & $E D^{b}$ & 2 & Hexaclorobenzeno & 0,010 & 0,0133 & 0,0001 & 0,000048 & $-0,000052$ \\
\hline NE33 & Faxineira & 3 & Hexaclorobenzeno & 0,010 & 0,0942 & 0,0001 & 0,000341 & 0,000241 \\
\hline
\end{tabular}


O composto organoclorado encontrado com maior incidência foi o heptacloro epóxi, presente em $43 \%$ das amostras. Apenas uma amostra, identificada por NE08, apresentou concentração do contaminante acima dos limites estabelecidos para IDA (Tabela 2).

Das amostras analisadas 13\% apresentaram resíduos de heptacloro. A amostra NE08 também apresentou resultados acima da IDA (Tabela 2) para este organoclorado.

A IDA estabelecida para resíduos de heptacloro e heptacloro epóxi é de $0,0001 \mathrm{mg} \mathrm{Kg}^{-1}$ de peso corporal, referindo-se à soma dos níveis toleráveis dos produtos. A amostra NE08 apresentou níveis acima dos aceitáveis para ambos os compostos (TID $=0,00031 \mathrm{mg}$ $\mathrm{kg}^{-1}$ de massa corporal), sendo este valor 3 vezes maior que o recomendado. A amostra E19 apresentou valor de $\mathrm{TID}=0,000123 \mathrm{mg} \mathrm{kg}^{-1}$ de massa corporal para heptacloro epóxi, 23\% acima do determinado pelo CODEX [9].

Os TID médios encontrados neste trabalho foram de $0,00059 \mathrm{mg} \mathrm{kg}^{-1}$ de massa corporal para $\mathrm{o}$ heptacloro epóxi e $0,00075 \mathrm{mg} \mathrm{kg}^{-1}$ de massa corporal para o heptacloro, valores correspondentes à concentração média de 0,001637 e $0,001971 \mathrm{mg} \mathrm{kg}^{-1}$, respectivamente. Estes valores são muito próximos aos encontrados por Matuo et al. [10], que estudou resíduos de organoclorados em amostras de leite humano da região de Ribeirão Preto, SP, encontrando heptacloro em 66\% das 37 amostras analisadas, com concentração média de $0,0014 \mathrm{mg} \mathrm{kg}^{-1}$.

O dieldrin foi detectado em $30 \%$ das amostras avaliadas. Das amostras analisadas, somente as identificadas como NE25 e NE26 apresentaram valores acima dos estabelecidos pela legislação vigente; nas demais, os níveis detectados estiveram abaixo do LMRE (Tabela 2).

Os organoclorados aldrin e o endrin não foram detectados nas amostras analisadas. O aldrin, após a absorção, sofre rápida metabolização hepática, transformando-se em seu epóxido correspondente, o dieldrin. Deste modo, caso não hajam indícios de exposição recente ao composto, existe pouca probabilidade de se encontrar este resíduo em sua forma original $[8,17]$.

Em 18\% das amostras de leite foram encontrados resíduos de hexaclorobenzeno, sendo que, duas amostras apresentaram concentração acima do LMRE permitido para este composto (E36 e NE33). A amostra E36 apresentou valor de $276 \%$ acima do LMRE, enquanto que os resultados da amostra NE33 foram $842 \%$ acima do permitido. Para estas duas amostras,
A TID do Hexaclorobenzeno foi respectivamente 36 e $241 \%$ maiores que a IDA determinada pelo CODEX. Não foi possível explicar porque estas amostras apresentaram resíduo de hexaclorobenzeno em níveis considerados elevados. Alguns autores atribuem este fato a contaminações originárias de produtos empregados em uso doméstico ou acidental [18]. Segundo Cunha [19], um dos principais usos do HCB técnico foi na atividade agrícola, florestal e para fins sanitários, inclusive no uso doméstico para controle de escabiose.

Em $5 \%$ das amostras analisadas, foram encontrados p,p' DDE e o,p' DDE, respectivamente, e em 3\% o organoclorado metoxicloro. Segundo os critérios estabelecidos pela FAO, os níveis residuais destes compostos foram considerados toleráveis (abaixo da LMRE). Entretanto, as concentrações presentes estiveram acima do limite de detecção do método empregado, sendo que, num alimento importante como o leite materno, seria desejável uma completa ausência de contaminação por este tipo de composto. O organoclorado lindane foi detectado em 5\% das amostras analisadas, entretanto todas abaixo dos limites seguros estabelecidos para este pesticida. Do total de amostras analisadas, 35\% não apresentaram níveis detectáveis dos pesticidas organoclorados avaliados.

\section{Discussão}

Em doadoras de leite materno de Cuiabá, MT, $12 \%$ das amostras de leite estavam contaminadas por heptacloro [20]. Do total de 32 doadoras pesquisadas, 4 amostras de leite apresentaram resíduos de heptacloro, porém, todas com níveis abaixo do determinado pelo LMRE.

Em estudos realizados com leite humano em diferentes regiões [9, 21-26] pode-se observar que, devido a grande diversidade de organoclorados utilizados e a variedade de culturas e costumes, não é possível traçar um perfil epidemiológico ou avaliar os resíduos de organoclorados detectados, ao longo dos anos. O perfil tem um caráter regional, muito ligado ao tipo de agricultura que se praticou e da cultura predominante na região. Ou seja, há uma clara necessidade de investigações detalhadas e locais quanto aos níveis de organoclorados presentes em amostras de leite humano, sendo igualmente importante se conhecer as principais vias de exposição a estes contaminantes.

$\mathrm{O}$ aldrin foi um pesticida muito utilizado na agricultura, principalmente em regiões canavieiras, como 
é o caso de Piracicaba, SP. Os organoclorados heptacloro e aldrin, além de serem muito empregados como formicidas, são também recomendados para outras culturas. Em levantamentos feitos até 1983, o dieldrin chegou a ser detectado em $100 \%$ das amostras de leite humano, sendo que, em 1992, foi encontrado com freqüência média de 20\%, [10]. Na Suécia, as concentrações de dieldrin encontradas no leite humano em 1985 corresponderam a 13\% do encontrado em 1967 [18].

No Brasil são conhecidos poucos casos de contaminação pelo hexaclorobenzeno, entre eles o ocorrido no município de Duque de Caxias-RJ, onde foi instalada uma unidade de produção na década de 50. A fábrica foi desativada em 1960, porém, estudos desenvolvidos pela Fundação Estadual de Engenharia de Meio Ambiente do Rio de janeiro indicaram a existência de aproximadamente $15.000 \mathrm{~m}^{3}$ de solo contaminado na região, com concentração média superior a $100 \mathrm{mg} \mathrm{kg}$ ${ }^{-1}$. A região de São Caetano do Sul-SP vem enfrentando problema semelhante, onde se instalou o complexo industrial Matarazzo, desativado na década de 80 . Na avaliação do risco de contaminação realizada por Cunha [19] nessas áreas contaminadas por fontes industriais desativadas, os resultados apresentaram elevados níveis de contaminação no solo.

Em países mais desenvolvidos, a proibição de organoclorados como hexaclorobenzeno e lindane tem resultado em um declínio gradual dos níveis destas substâncias no ambiente. No monitoramento realizado na Suécia, não foi observado declínio acentuado destes resíduos até 1974, sendo que a partir desta data as concentrações de hexaclorobenzeno foram gradativamente diminuindo e, em 1997, representavam apenas 5\% dos valores originalmente determinados para 1974 [18].

Em outros estudos, porém, apesar da tendência de declínio verificada, o mesmo foi mais lento, sendo que as concentrações encontradas parecem refletir a circulação total dessas substâncias em todo o planeta. Em duas cidades da Espanha, Granada e Alméria, Campoy et al. [27] obtiveram como resultados preliminares $94 \%$ das amostras de leite humano contaminadas com HCB, o que se justifica pelo fato do HCB ter sido muito utilizado como fungicida até 1980 em toda a Europa.

A presença de resíduos de organoclorados em leite materno é relatada por diversos autores em países como Austrália [3], Argentina [4], Índia [28] e China [26] e um decréscimo não tão acentuado dessas concentrações tem sido observada nas últimas décadas. Essa desaceleração pode estar relacionada à ingestão de alimentos oriundos de regiões onde os organoclorados são permitidos. No Brasil, apesar da proibição do uso de organoclorados desde 1985 seu uso clandestino ainda é representativo, seu uso é permitido em campanhas públicas para combate de vetores transmissores de malária e em outros países são utilizados nas plantações de tabaco. Atualmente o organoclorado dicofol é permitido nas culturas de algodão, citros e maça e o organoclorado endosulfam, classificado pelo Ministério da Agricultura como ciclodienoclorado, ainda é permitido nas culturas de algodão, cacau, café, cana-de-açúcar e soja.

No Brasil, o primeiro trabalho avaliando resíduos de DDT foi apresentado por Matuo [8], em Ribeirão Preto, SP. Passado uma década, Matuo [29] observou que os níveis de DDT encontrado na mesma região haviam diminuído.

O DDT e seus metabólitos foram os organoclorados que mais despertaram interesse dos pesquisadores. Os primeiros alertas sobre o DDT tiveram início nos anos sessenta, do século passado, quando alguns ambientalistas já discutiam sobre alterações observadas no meio ambiente. Após a proibição do uso nos anos 70 estudos de monitoramento têm apresentado o DDT como o composto que vêm apresentando maior queda nas concentrações. Na Suécia [18], pesquisas mostraram que, em 1997 a concentração de DDT representava 1\% da encontrada em 1967. O p,p' DDE avaliado a partir de 1972 apresentou um decréscimo de 95\% na avaliação realizada em 1997.

\section{Conclusão}

Resíduos de organoclorados foram encontrados em $65 \%$ das amostras de leite humano avaliadas sendo detectados, principalmente, os compostos: heptacloro epóxi, heptacloro, dieldrin e hexaclorobenzeno. Em apenas 1 doadora, a IDA apresentou-se acima do limite estabelecido pela FAO/WHO. O contaminante mais freqüente neste grupo de doadoras foi o heptacloro epóxi, presente em $43 \%$ do leite das doadoras. Isto reflete a realidade da região de Piracicaba, SP, onde a cultura de cana-de-açúcar é economicamente importante e, onde este organoclorado foi utilizado em larga escala.

Quanto ao hexaclorobenzeno, encontrado em $18 \%$ das doadoras, o fato de uma doadora apresentar nível 3,4 vezes acima do limite estabelecidos pelos órgãos ligados a saúde foi tratado como um caso isolado, pois, pelos dados levantados, não foi possível estabelecer as origens desta contaminação. 
Evidencia-se a necessidade de maiores estudos sobre os organoclorados na região, evitando-se o agravamento do problema. Faz-se necessário ainda maiores esforços para o estabelecimento de programas de educação voltados à comunidade e medidas de prevenção de riscos à saúde humana e ao ambiente.

Diante dos níveis de contaminação e das vantagens associadas ao aleitamento materno, este estudo se apresenta como um alerta às autoridades sobre a presença de contaminantes que, apesar de dentro dos limites, não deveriam estar presentes no leite humano.

\section{Agradecimentos}

Os autores agradecem à Fundação de Amaro à Pesquisa do Estado de São Paulo e ao Conselho Nacional de Desenvolvimento Científico e Tecnológico pelo apoio financeiro.

\section{Referências:}

[1] S. H. G. Brondi, Tese (Doutorado) - Instituto de Química de São Carlos, Universidade de São Paulo, (2000) 133.

[2]. C. A. Harris, M. W. Woolridge, A. W. M. Hay, Chemosphere 43 (2001) 243.

[3]. J. F. Mueller, F. Harden, L. M. Toms, R. Symons, P.

Fürst, Chemosphere 70 (2008) 712.

[4]. S. der Parsehian, Rev. Hosp. Mat. Inf. Ramón Sardá 27 (2) (2008) 70.

[5]. E. P. Laug, F. M. Kunze, E. S. Prickett, Arch. Ind. Hyg. 3 (1951) 245.

[6]. Y. K. Matuo, J. N. C. Lopes, J. L. C. Lopes, Rev. Bras. Biol. 40 (20) (1990) 293.

[7]. BRASIL. Ministério da Agricultura. Portaria no 329-MA, (1985) 1.

[8]. Y.K. Matuo, Dissertação (Mestrado) - Escola de Enfermagem, Universidade de São Paulo. (1978) 71.

[9]. FAO/WHO, Codex Alimentarius, (1996). v.2B.

[10] Y. K. Matuo, J. N. C. Lopes, I. C. Casanova, T. Matuo, J. L. C Lopes, Arch. Environ. Contam. Toxicol. 22 (1992) 167.

[11]. World Health Organization. (1987) 70.

[12]. ANVISA. Disponível em: <http://www.anvisa. gov.br/ALIMENTOS/informes

/17_190106.htm>.
Acessado em 14/agos/2009.

[13]. J. Mañes, G. Font, Y. Picó, J. Chromat. 642 (1993) 195.

[14]. D. G. Kelts; E. G. Jones, Manual de nutrição infantil. Rio de Janeiro: Editora Guanabara, 1995, 312.

[15]. INMETRO. Orientação sobre validação de métodos de ensaios químicos: DOQ-CGCRE-008. Rev. 02, (2007) 24.

[16]. M. Ribani, C. B. G. Bottoli, C. H. Collins, I. C. S. F. Jardim, L. F. C. MELO, Quím. Nova 27(5) (2004) 771.

[17]. K. Suassuna, Brasil:Green peace, (2001) 17.

[18]. K. Norén; D. Meironyté, Chemosphere 40 (2000) 1111.

[19]. R. C. A. Cunha, Tese (Doutorado) - Instituto de Geociências, Universidade de São Paulo, (1997) 151.

[20]. M. A. G. Oliveira, E. F. G. Dores-Carvalho, Rev. Ecotoxicol. e Meio Ambiente 8 (1998) 77.

[21]. L. S. Sant' Ana, Dissertação (Mestrado) - Faculdade de Farmácia, Universidade Federal de Minas Gerais (1988) 118.

[22]. J. L. Mello, Dissertação (Mestrado) - Escola Nacional de Saúde Pública (1999) 127.

[23]. S. A. Mesquita, Dissertação (Mestrado) - Escola Nacional de Saúde Pública (2001) 74.

[24]. M. A. G. Oliveira, E. F. G. Dores-Carvalho, Rev. Ecotoxicol. e Meio Ambiente 8 (1997) 77.

[25]. R. D. Behrooz, A. E. Sari, N. Bahramifar, S. M. Ghasempouri, Chemosphere 74 (2009) 931.

[26]. H. R. Chao, S. L. Wang, T. C. Lin, X. H. Chung, Chemosphere 62 (2006) 1774.

[27]. C. Campoy, et al. Early Hum. Dev. 65 (2001) 173. [28]. A. Kumar, A. Baroth, I. Soni, P. Bhatnagar, P. J. John, Env. Monitoring and Assessment 116 (2006) 1. [29]. Y. K. MATUO, Tese (Doutorado) - Escola de Enfermagem, Universidade de São Paulo (1987) 189. 